\title{
THE STRAIN OF A NON-GRAVITATING SPHERE OF VARIABLE
}

\section{DENSITY*}

\author{
BY \\ L. M. HOSKINS \\ §1. Introductory.
}

The mathematical problem of the strain of an elastic sphere is of special physical interest because of its relation to the question of the elastic yielding of the earth to disturbing forces. The cases of the problem which have been solved, however, involve assumptions which depart from what is known or thought probable in the case of the earth. The solutions of LAME and KELviN apply to a bomogeneous body acted upon by bodily forces which are known functions of the coördinates. This case disagrees with the facts as regards the earth in two particulars: (1) The earth is certainly far from homogeneous in density and is very likely far from homogeneous in elastic properties. (2) The important bodily forces of self-gravitation cannot be expressed as functions of the coördinates in advance of the solution, since the changes in these forces caused by the strain are of the same order of importance as the external disturbing forces; it is only by assuming incompressibility that it becomes possible to express the gravitational forces without the use of unknown functions.

In a previous paper $\dagger$ was given a solution of the problem for a compressible sphere initially of uniform density, taking account of the changes in the gravitational forces caused by the strain.

In the present paper will be found a solution for the case of a non-gravitating sphere of which the density is a given function of the distance from the center, the elastic moduli being assumed to be uniform in value throughout the body.

By combining these two cases, and introducing the additional assumption that the elastic moduli are functions of the distance from the center, we should have perhaps the nearest possible approximation to a completely general statement of the problem presented by the actual earth. While there is no difficulty in forming the differential equations for this general problem in a manner analogous to that employed in the two cases which have been solved, a solution of these general equations has not thus far been obtained.

\footnotetext{
* Presented to the Society, San Francisco Section, February 26, 1910.

†Transactions of the American Mathematical Society, vol. 11 (1910), p. 203. 
The case treated in this paper appears, however, to possess some interest and importance, being a generalization in one important particular of the problem which formed the basis of the original estimates of the rigidity of the earth.

\section{§ 2. Statement of Problem.}

The problem here treated is the following:

To determine the strain of an elastic sphere of which the density is a given function of the distance from the center, under the action of bodily forces having a potential, but free from stress on the bounding surface. The potential may be any function which, when expressed in polar coördinates, is developable as to $\theta$ and $\phi$ in a series of spherical surface harmonics.

\section{§3. Equations of Equilibrium of Volume-Element.}

Using polar coördinates $r, \theta, \phi$, let $u_{r}, u_{\theta}, u_{\phi}$ be the elastic displacenents, $\varpi_{r}, \varpi_{\theta}, \varpi_{\phi}$ the rotation-components, $\Delta$ the cubical dilatation, $F_{r}, F_{\theta}, F_{\phi}$ the components of bodily force per unit mass, $\lambda+\frac{2}{3} \mu, \mu$ the bulk-modulus and rigidity-modulus, $\rho$ the density.

The three equations of equilibrium for an element of volume are the following:

in which

$$
\begin{aligned}
& (\lambda+2 \mu) \frac{\partial \Delta}{\partial r}-\frac{2 \mu}{r \sin \theta}\left[\frac{\partial}{\partial \theta}\left(\varpi_{\phi} \sin \theta\right)-\frac{\partial \varpi_{\phi}}{\partial \phi}\right]+\rho F_{r}=0, \\
& (\lambda+2 \mu) \frac{1}{r} \frac{\partial \Delta}{\partial \theta}-\frac{2 \mu}{r \sin \theta}\left[\frac{\partial \varpi_{r}}{\partial \dot{\phi}}-\frac{\partial}{\partial r}\left(r \varpi_{\phi} \sin \theta\right)\right]+\rho F_{\theta}=0, \\
& (\lambda+2 \mu) \frac{1}{r \sin \theta} \frac{\partial \Delta}{\partial \phi}-\frac{2 \mu}{r}\left[\frac{\partial}{\partial r}\left(r \varpi_{\theta}\right)-\frac{\partial \varpi_{r}}{\partial \theta}\right]+\rho F_{\phi}=0 ;
\end{aligned}
$$

$$
\Delta=\frac{1}{r^{2} \sin \theta}\left[\frac{\partial}{\partial r}\left(r^{2} u_{r} \sin \theta\right)+\frac{\partial}{\partial \theta}\left(r u_{\theta} \sin \theta\right)+\frac{\partial}{\partial \phi}\left(r u_{\phi}\right)\right]
$$

$$
\begin{aligned}
2 \varpi_{r} & =\frac{1}{r^{2} \sin \theta}\left[\frac{\partial}{\partial \theta}\left(r u_{\phi} \sin \theta\right)-\frac{\partial}{\partial \phi}\left(r u_{\theta}\right)\right], \\
2 \varpi_{\theta} & =\frac{1}{r \sin \theta}\left[\frac{\partial u_{r}}{\partial \phi}-\frac{\partial}{\partial r}\left(r u_{\phi} \sin \theta\right)\right], \\
2 \varpi_{\phi} & =\frac{1}{r}\left[\frac{\partial}{\partial r}\left(r u_{\theta}\right)-\frac{\partial u_{r}}{\partial \theta}\right] .
\end{aligned}
$$

Let $W$ denote the potential of the bodily forces, and assume that

$$
W=\Sigma R_{i} S_{i}
$$


in which $S_{i}$ denotes a spherical surface harmonic of order $i$, and $R_{i}$ a function of $r$. Then

$$
\begin{aligned}
& F_{r}=\Sigma \frac{d R_{i}}{d r} S_{i}, \\
& F_{\theta}=\Sigma \frac{R_{i}}{r} \frac{\partial S_{i}}{\partial \theta}, \\
& F_{\phi}=\Sigma \frac{R_{i}}{r \sin \theta} \partial S_{i}
\end{aligned}
$$

A solution of equations (1), (2) and (3) may be formed by putting together solutions corresponding to the separate terms in the value of $W$. We therefore confine the following discussion to the solution corresponding to a single term $R_{i} S_{i}$.

We now assume

$$
u_{r}=u S_{i}^{\vee}, \quad u_{\theta}=v \frac{\partial S_{i}}{\partial \theta}, \quad u_{\phi}=\frac{v}{\sin \theta} \frac{\partial S_{i}}{\partial \phi},
$$

in which $u$ and $v$ are functions of $r$ only. Substituting these values in equations $(4), \cdots,(7)$, and making use of the differential equation

$$
\frac{1}{\sin ^{2} \theta} \frac{\partial^{2} S_{i}}{\partial \phi^{2}}+\frac{1}{\sin \theta} \frac{\partial}{\partial \theta}\left(\sin \theta \frac{\partial S_{i}}{\partial \theta}\right)+i(i+1) S_{i}=0
$$

which is satisfied by any spherical surface harmonic of order $i$, we find

$$
\begin{aligned}
& \Delta=\left[\frac{1}{r^{2}} \frac{d\left(r^{2} u\right)}{d r}-i(i+1) \frac{v}{r}\right] S_{i}, \\
& 2 \sigma_{r}=0 \text {, } \\
& 2 \varpi_{\theta}=\frac{1}{r}\left[u-\frac{d(r v)}{d r}\right] \frac{1}{\sin \theta} \frac{\partial S_{i}}{\partial \phi}, \\
& 2 \varpi_{\phi}=-\frac{1}{r}\left[u-\frac{d(r v)}{d r}\right] \frac{\partial S_{i}}{\partial \theta} .
\end{aligned}
$$

If the foregoing values of $F_{r}, F_{\theta}, F_{\phi}, \Delta, \varpi_{r}, \varpi_{\theta}$ and $\varpi_{\phi}$ be substituted in $(1),(2)$ and (3), these are freed from $\theta$ and $\phi$. Equations (2) and (3) in fact become identical, and there remain to be satisfied two ordinary differential equations for determining $u, v$ as functions of $r$. Writing $R$ for $R_{i}$, these equations are the following:

$$
\begin{aligned}
(\lambda+2 \mu) \frac{d}{d r}\left(\frac{1}{r^{2}} \frac{d\left(r^{2} u\right)}{d r}-i(i\right. & \left.+1) \frac{v}{r}\right) \\
& +\frac{i(i+1) \mu}{r^{2}}\left(\frac{d(r v)}{d r}-j+\rho \frac{d R}{d r}=0,\right.
\end{aligned}
$$




$$
\left.(\lambda+2 \mu)\left(\begin{array}{c}
1 d\left(r^{2} u\right) \\
r^{2} d r
\end{array}-i(i+1){ }_{r}^{v}\right)+\mu \frac{d}{d i}\left(\begin{array}{c}
d(r v) \\
d r
\end{array}\right)+u\right)+\rho R \doteq 0
$$

It is now convenient to replace $r$ by $x=r / a$ as independent variable, and to introduce $y$ and $z$, defined by the equations

$$
\begin{aligned}
& y=\frac{\Delta}{S_{i}}=\frac{1 d\left(r^{2} u\right)}{r^{2}}-i(i+1) \frac{v}{r}, \\
& z=\underset{\partial S_{i} / \partial \theta}{2 \varpi_{\phi}}=\frac{1}{r}\left(\frac{d(r v)}{d r}-u\right),
\end{aligned}
$$

thus reducing the differential equations to the following:

$$
\begin{gathered}
(\lambda+2 \mu) \frac{d y}{d x}+i(i+1) \mu \frac{z}{x}+\rho \frac{d R}{d x}=0, \\
(\lambda+2 \mu) y+\mu \frac{d(x z)}{d x}+\rho R=0 .
\end{gathered}
$$

\section{§4. Surface Conditions.}

We now have to express the condition that the bounding surface is free from stress, that is,

$$
\widehat{r r}=0, \quad \widehat{r} \theta=0, \quad \widehat{r \phi}=0
$$

Using (10) in the general formulas for these stresses, we find

$$
\begin{aligned}
& \widehat{r r}=\lambda \Delta+2 \mu \frac{\partial u_{r}}{\partial r}=\left(\lambda y+2 \mu \frac{d u}{d r}\right) S_{i}, \\
& \widehat{r \theta}=\mu\left(\frac{\partial u_{\theta}}{\partial r^{\prime}}-\frac{u_{\theta}}{r}+\frac{1}{r} \frac{\partial u_{r}}{\partial \theta}\right)=\mu\left(\frac{d v}{d r}-\frac{v}{r}+\frac{u}{r}\right) \frac{\partial S_{i}}{\partial \theta}, \\
& \widehat{r \phi}=\mu\left(\begin{array}{cc}
1 & \partial u_{r} \\
r \cdot \sin \theta & \partial \phi
\end{array}+\frac{\partial u_{\phi}}{\partial r}-\frac{u_{\phi}}{r}\right)=\mu\left(\frac{d v}{d r}-\frac{v}{r}+\frac{u}{r}\right) \frac{1}{\sin \theta \partial \phi},
\end{aligned}
$$

so that equations (22) reduce to the two

$$
\begin{aligned}
& \lambda y+2 \mu \frac{d u}{d r}=0, \\
& \frac{d v}{d r}-\frac{v}{r}+\frac{u}{r}=0,
\end{aligned}
$$

to be satisfied when $x=1$. 


\section{§5. Solution of Differential Equations.}

By eliminating successively $z$ and $y$ between equations (20) and (21) we obtain the two equations

$$
\begin{gathered}
\left(x^{2} \frac{d^{2}}{d x^{2}}+2 x \frac{d}{d x}-i(i+1)\right)((\lambda+2 \mu) y+\rho R)-\frac{d}{d x}\left(x^{2} R \frac{d \rho}{d x}\right)=0 \\
\mu\left(x^{2} \frac{d^{2} z}{d x^{2}}+2 x \frac{d z}{d x}-i(i+1) z\right)+x R \frac{d \rho}{d x}=0
\end{gathered}
$$

The solutions of these equations are the following:

$$
\begin{aligned}
(2 i+1)[(\lambda+2 \mu) y+\rho R]=x^{i} \int x^{-i-1} & \frac{d}{d x}\left(x^{2} R \frac{d \rho}{d x}\right) d x \\
& -x^{-i-1} \int x^{i} \frac{d}{d x}\left(x^{2} R \frac{d \rho}{d x}\right) d x,
\end{aligned}
$$

$$
(2 i+1) \mu z=-x^{i} \int x^{-i-1}\left(x R \frac{d \rho}{d x}\right) d x+x^{-i-1} \int x^{i}\left(x R \frac{d \rho}{d x}\right) d x
$$

in which, for the general solutions, each integral must be understood to involve an arbitrary constant. These constants are here omitted, since they are accounted for in the complementary solutions to be obtained presently. Modifying by integration by parts, we find

$$
\begin{gathered}
(\lambda+2 \mu) y=-\frac{1}{2 i+1}\left[(i+1) x^{i} \int \rho \frac{d}{d x}\left(\frac{R}{x^{i}}\right) d x+\frac{i}{x^{i+1}} \int \rho \frac{d\left(R x^{i+1}\right)}{d x} d x\right] \\
\mu z=\frac{1}{2 i+1}\left[x^{i} \int \rho \frac{d}{d x}\left(\frac{R}{x^{i}}\right) d x-\frac{1}{x^{i+1}} \int \rho \frac{d\left(R x^{i+1}\right)}{d x} d x\right] .
\end{gathered}
$$

These values of $y$ and $z$ may now be substituted in (18) and (19), giving two simultaneous linear differential equations for determining $u$ and $v$. The solutions of these equations may conveniently be expressed as follows :

From (18) and (19) we readily obtain the equations

$$
\frac{d}{d x}(i v-u)+\frac{i+2}{x}(i v-u)=a(i z-y),
$$

$$
\frac{d}{d x}[(i+1) v+u]-\frac{i-1}{x}[(i+1) v+u]=a[(i+1) z+y]
$$

of which the solutions are

$$
i v-u=\frac{a}{x^{i+2}} \int(i z-y): c^{i+2} d x,
$$




$$
(i+1) v+u=a x^{i-1} \int \frac{(i+1) z+y}{x^{i-1}} d x .
$$

These are easily solved for $u$ and $v$.

The forms of the complementary solutions are easily seen by considering the constants of integration in (32), (33), (36) and (37). The values of the constants must be determined so as to satisfy $(18),(19),(20)$ and (21). The complementary solutions are thus found to be the following:

$$
\begin{aligned}
y & =C_{i} x^{i}+C_{-i-1} x^{-i-1}, \\
z & =D_{i} x^{i}+D_{-i-1} x^{-i-1}, \\
\frac{u}{r} & =A_{i} x^{i}+A_{-i-1} x^{-i-1}+A_{i-2} x^{i-2}+A_{-i-3} x^{-i-3}, \\
\frac{v}{r} & =B_{i} x^{i}+B_{-i-1} x^{-i-1}+B_{i-2} x^{i-2}+B_{-i-3} x^{-i-3} ;
\end{aligned}
$$

in which the twelve constants must satisfy the eight equations

$$
\begin{aligned}
(\lambda+2 \mu) C_{i}+(i+1) \mu D_{i} & =0, \\
(i+3) A_{i}-i(i+1) B_{i}-C_{i} & =0, \\
A_{i}-(i+2) B_{i}+D_{i} & =0, \\
(\lambda+2 \mu) C_{-i-1}-i \mu D_{-i-1} & =0, \\
(i-2) A_{-i-1}+i(i+1) B_{-i-1}+C_{-i-1} & =0, \\
A_{-i-1}+(i-1) B_{-i-1}+D_{-i-1} & =0, \\
A_{i-2}-i B_{i-2} & =0, \\
A_{-i-3}+(i+1) B_{-i-3} & =0 .
\end{aligned}
$$

The complete solutions will thus involve four arbitrary constants; by assigning values to these we may satisfy four equations of condition at the boundary.

This solution covers the case of a spherical shell with bounding surfaces free from stress, or with arbitrary surface stress of a restricted type (corresponding to the restriction imposed on the potential of the bodily forces), or with arbitrary surface displacements of a similarly restricted type.

For the case of the complete sphere, negative powers of $x$ must be rejected, so that the only non-vanishing coefficients in (38) are $A_{i-2}, A_{i}, B_{i-2}, B_{i}, C_{i}$, $D_{i}$; these being connected by four relations included among (39). There remain two arbitrary constants for satisfying the surface conditions (26) and (27). 
§ 6. Case in which the Potential is a Harmonic Function.

If $W$ is a harmonic function, each term $R_{i} S_{i}$ in its development must satisfy Laplace's equation, so that

$$
R_{i}=A r^{i}+B r^{-i-1} \text {. }
$$

Rejecting the negative power, we assume $R_{i}$ or $R$ to have the form $A v^{i}$. Equations (32) and (33) then reduce to

$$
\begin{aligned}
(\lambda+2 \mu) y & =-\frac{i}{(2 i+1) x^{i+1}} \int \rho \frac{d\left(R x^{i+1}\right)}{d x} d x, \\
\mu z & =-\frac{1}{(2 i+1) x^{i+1}} \int \rho \frac{d\left(R x^{i+1}\right)}{d x} d x .
\end{aligned}
$$

The further discussion will be restricted to the case in which $i=2$.

\section{\$7. Sphere Strained by Centrifugal Force.}

If the bodily forces are the centrifugal forces due to uniform rotation about a fixed axis, we have

$$
W=-\frac{c g}{3 a} r^{2} P_{2}
$$

in which $c$ denotes the ratio of equatorial centrifugal force to gravity, and $P_{2}$ is the zonal harmonic $\frac{3}{2} \cos ^{2} \theta-\frac{1}{2}$. We now take

$$
\begin{aligned}
& S_{2}=-\frac{2}{3} P_{2}=\frac{1}{8}-\cos ^{2} \theta, \\
& R_{2}=R=\frac{c g r^{2}}{2 a}=\frac{c g a}{2} x^{2} .
\end{aligned}
$$

In accordance with the notation of a former paper," let $e$ denote the ellipicicity of a surface originally spherical and of radius $r$, and $\alpha$ the angular displacement of a radius vector for which $\theta=45^{\circ}$. With the above value of $S_{2}$, we have

$$
e=\frac{u}{r}, \quad \alpha=\frac{v}{r} .
$$

In writing the complete solutions of the differential equations for this case, we take $A_{0}$ and $A_{2}$ as arbitrary constants, expressing $B_{0}, B_{2}, C_{2}, D_{2}$ in terms of them by means of (39). We thus obtain the equations

$$
y=-\frac{7 \mu}{\lambda} A_{2} x^{2}-\frac{c g a}{(\lambda+2 \mu) x^{3}} \int_{0}^{x} \rho x^{4} d x,
$$

\footnotetext{
*Transactions American Mathematical Society, vol. 11 (1910), p. 206.
} 
1910]

A SPHERE OF VARIABLE DENSITY

501

$$
\begin{gathered}
z=\frac{7(\lambda+2 \mu)}{3 \lambda} A_{2} x^{2}-\frac{c g a}{2 \mu x^{3}} \int_{0}^{x} \rho x^{4} d x \\
e=A_{0}+A_{2} x^{2}+\frac{c g a}{30 \mu(\lambda+2 \mu)}\left[\frac{5(3 \lambda+5 \mu)}{x^{3}} \int_{0}^{x} \rho x^{4} d x\right. \\
\left.\quad-\frac{9(\lambda+\mu)}{x^{5}} \int_{0}^{x} \rho x^{6} d x-2(3 \lambda+8 \mu) \int_{0}^{x} \rho x d x\right], \\
\alpha=\frac{1}{2} A_{0}+\frac{5 \lambda+7 \mu}{6 \lambda} A_{2} x^{2} \quad \frac{c g a}{30 \mu(\lambda+2 \mu)}\left[\frac{5 \mu}{x^{3}} \int_{0}^{x} \rho x^{4} d x+\frac{3(\lambda+\mu)}{x^{5}} \int_{0}^{x} \rho x^{6} d x-(3 \lambda+8 \mu) \int_{0}^{x} \rho x d x\right] .
\end{gathered}
$$

Substituting these values in (26) and 27), we obtain the following equations for determining $A_{0}$ and $A_{2}$ :

$$
\begin{aligned}
2 A_{0}-A_{2}+\frac{c g a}{30 \mu(\lambda+2 \mu)} & {\left[-10(9 \lambda+10 \mu) \int_{0}^{1} \rho x^{4} d x\right.} \\
& \left.+72(\lambda+\mu) \int_{0}^{1} \rho x^{6} d x-4(3 \lambda+8 \mu) \int_{0}^{1} \rho x d x\right]=0 \\
A_{0}+\frac{8 \lambda+7 \mu}{3 \lambda} A_{2}+\frac{c g a}{30 \mu(\lambda+2 \mu)}\left[5(3 \lambda+2 \mu) \int_{0}^{1} \rho x^{4} d x\right. & {\left[24(\lambda+\mu) \int_{0}^{1} \rho x^{6} d x-2(3 \lambda+8 \mu) \int_{0}^{1} \rho x d x\right]=0 }
\end{aligned}
$$

Solving, we find

$$
\begin{aligned}
A_{0}= & \frac{c g a}{30 \mu(\lambda+2 \mu)(19 \lambda+14 \mu)}\left[25\left(27 \lambda^{2}+56 \lambda \mu+28 \mu^{2}\right) \int_{0}^{1} \rho x^{4} d x\right. \\
& \left.-504(\lambda+\mu)^{2} \int_{0}^{1} \rho x^{6} d x+2(3 \lambda+8 \mu)(19 \lambda+14 \mu) \int_{0}^{1} \rho x d x\right] .
\end{aligned}
$$

$$
A_{2}=\frac{c g a}{30 \mu(\lambda+2 \mu)(19 \lambda+14 \mu)} \cdot 360 \lambda(\lambda+\mu)\left(\int_{0}^{1} \rho x^{6} d x-\int_{0}^{1} \rho x^{4} d x\right) \text {. }
$$

Of especial interest are the surface values of $e$ and $\alpha$ :

$$
e_{1}=\frac{\operatorname{cg} a}{2 \mu(19 \lambda+14 \mu)}\left[5(8 \lambda+7 \mu) \int_{0}^{1} \rho x^{4} d x-21(\lambda+\mu) \int_{0}^{1} \rho x^{6} d x\right],
$$

$$
\alpha_{1}=\frac{c g a}{4 \mu(19 \lambda+14 \mu)}\left[5 \lambda \int_{0}^{1} \rho x^{4} d x+14(\lambda+\mu) \int_{0}^{1} \rho x^{6} d x\right] \text {. }
$$


If $\rho$ is constant it is easy to show that the foregoing equations give the following well-known results :

$$
\begin{aligned}
& e=\frac{c}{2(19 \lambda+14 \mu)}\left[2(4 \lambda+3 \mu)-(3 \lambda+2 \mu) x^{2}\right] \frac{\rho g a}{\mu}, \\
& \alpha=\frac{c}{4(19 \lambda+14 \mu)}\left[2(4 \lambda+3 \mu)-(5 \lambda+4 \mu) x^{2}\right] \frac{\rho g a}{\mu} .
\end{aligned}
$$

\section{§ 8. Laplace's Law of Density.}

To illustrate the application of the above formulas to a case of variable density, assume Laplace's law, expressed by the formula

$$
\rho=\rho_{0} \frac{\sin \kappa x}{\kappa x}=\frac{\rho_{m} \kappa^{3}}{3(\sin \kappa-\kappa \cos \kappa)} \cdot \frac{\sin \kappa x}{\kappa x},
$$

in which $\rho_{m}$ is the mean density and $\rho_{0}$ the value of $\rho$ at the center. We then find

(60) $\frac{1}{x^{3}} \int_{0}^{x} \rho x^{4} d x=\frac{\rho_{m} \kappa}{3(\sin \kappa-\kappa \cos \kappa)}\left[\frac{6-\kappa^{2} x^{2}}{\kappa^{2} x^{2}} \cos \kappa x-\frac{6-\kappa^{2} x^{2}}{\kappa^{3} x^{3}} \sin \kappa x\right]$,

(61) $\frac{1}{x^{5}} \int_{0}^{o x} \rho x^{6} d x=\frac{\rho_{m} \kappa}{3(\sin \kappa-\kappa \cos \kappa)}\left[\frac{120-60 \kappa^{2} x^{2}+5 \kappa^{4} x^{4}}{\kappa^{5} x^{5}} \sin \kappa x\right.$

$$
\left.-\frac{120-20 \kappa^{2} x^{2}+\kappa^{4} x^{4}}{\kappa^{4} x^{4}} \cos \kappa x\right]
$$

If the ratio of mean density to surface density is assumed to be 2 , we find $\kappa=2.461$, and

$$
\int_{0}^{1} \rho x^{4} d x=.168 \rho_{m}, \quad \int_{0}^{1} \rho x^{6} d x=.108 \rho_{m} .
$$

These values substituted in (54) and (55) give

$$
\begin{aligned}
& \frac{e_{1}}{c}=\frac{4.45 \lambda+3.61 \mu}{2(19 \lambda+14 \mu)} \cdot \frac{\rho_{m} g a}{\mu}, \\
& \frac{\alpha_{1}}{c}=\frac{2.35 \lambda+1.51 \mu}{4(19 \lambda+14 \mu)} \cdot \frac{\rho_{m} g a}{\mu} .
\end{aligned}
$$

Comparing with the surface values of $e$ and $\alpha$ for a sphere of uniform density $\boldsymbol{\rho}_{m}$, 


$$
\begin{aligned}
& \frac{e_{1}}{c}=\frac{5 \lambda+4 \mu}{2(19 \lambda+14 \mu)} \cdot \frac{\rho_{m} g a}{\mu}, \\
& \frac{\alpha_{1}}{c}=\frac{3 \lambda+2 \mu}{4(19 \lambda+14 \mu)} \cdot \frac{\rho_{m} g a}{\mu},
\end{aligned}
$$

it appears that the assumption of variable density decreases $e_{1}$ by about 11 per cent and $\alpha_{1}$ by about 22 per cent.

We may also compare the values at the center, which with Laplace's law of density are found to be

$$
\frac{2 \alpha_{0}}{c}=\frac{e_{0}}{c}=\frac{A_{0}}{c}=\frac{124.4 \lambda^{2}+349.1 \lambda \mu+191.8 \mu^{3}}{30(\lambda+2 \mu)(19 \lambda+14 \mu)} \cdot \frac{\rho_{m} g a}{\mu},
$$

while the corresponding values for uniform density $\rho_{m}$ are

$$
\frac{2 \alpha_{0}}{c}=\frac{e_{0}}{c}=\frac{4 \lambda+3 \mu}{19 \lambda+14 \mu} \frac{\rho_{m} g a}{\mu}=\frac{120 \lambda^{2}+330 \lambda \mu+180 \mu^{2}}{30(\lambda+2 \mu)(19 \lambda+14 \mu)} \cdot \frac{\rho_{m} g a}{\mu} .
$$

The central values of $e$ and $\alpha$ are thus slightly increased by the assumption of variable density.

\section{§ 9. Gravitating Sphere.}

The results of the above solution cannot be applied directly to a gravitating body of size and mass comparable with those of the earth, since the changes in the gravitational forces caused by the strain will be of the same order of importance as the disturbing forces. There is no difficulty in expressing the gravitational forces in terms of the strain and the density and thus forming the differential equations which must replace (16) and (17) above given, but the solution of these equations appears to involve serious analytical difficulties.*

* In expressing the equations of interior equilibrium in terms of the strain, for the case of a gravitating body, it is necessary to assume that the changes of stress and strain from their initial values are related in the same way as if the initial condition were one of zero stress and strain. This assumption is the basis of the solution given for the case of a compressible sphere in the paper cited above, the reasoning being in this respect substantially identical with that previously applied to the case of incompressibility. (See A. E. H. LovE, $A$ Treatise on the Mathematical Theory of Elasticity, $2 \mathrm{~d}$ ed., p. 249.) It has sometimes been assumed that this reasoning is valid only in the case of incompressibility. Thus Love states that if the sphere is homogeneous and incompressible, the initial stress may be taken to be of the nature of a hydrostatio pressure, its value being then easily determined, and the terms which correspond to this initial stress in the equations of equilibrium being found to cancel with those representing the initial body-force. It seems clear, however, first that even in an incompressible body the initial condition may involve tangential stress, and secondly that the assumption of no initial tangential stress is not essential to the argument. Whatever values the initial stress-components may have, the terms representing them in the equations of equilibrium will cancel the terms representing the initial body-forces. 
If the body is assumed incompressible and of uniform density, the effect of gravitation can be taken account of synthetically by a method employed by Kelvin.* This method consists in combining the results for the two cases of a gravitating fluid and a non-gravitating elastic solid. It does not appear that similar reasoning can be applied if either of the assumptions of incompressibility and uniform density is dropped, since the distribution of the forces of gravitation cannot be assumed to be the same for the strained elastic solid as for the fluid.

The method of applying this principle in the solutions cited above, both for the case of incompressibility and for that of compressibility, consists in comparing the forces acting upon a volume-element in the strained condition with those initially acting upon the same volume-element. It would perhaps be more reasonable to deal with an individual element of matter, using the increments of the stress-components acting on that element in expressing the stress-strain relations by the usual formulas. The increment of stress for an individual element would be in equilibrium with the increment of body-force acting upon that element, which would consist in part of the change in the gravitational force due to the displacement of the attracted element. This would introduce additional terms into the equations of equilibrium, but would add no difficulty to their solution.

* Thomson and TarT's Natural Philosophy, § 840, Ed. of 1890. 